\title{
Ultimate squeezing through coherent quantum feedback
}

\author{
Alfred Harwood $\odot$ and Alessio Serafini $\odot$ \\ Department of Physics and Astronomy, University College London, Gower Street, WC1E 6BT, London, United Kingdom
}

(Received 21 October 2019; accepted 10 September 2020; published 20 October 2020)

\begin{abstract}
Coherent feedback is the use of feedback loops not involving any measurements for the purpose of quantum control. This can be contrasted to measurement-based feedback where the feedback signal is directed by the result of measurements upon the system. Here, we develop a general framework to describe interferometric coherent feedback loops and prove that, under any such scheme, the steady-state squeezing of a bosonic mode subject to a rotating wave coupling with a white-noise environment and to any quadratic Hamiltonian must abide by a noise-dependent bound that reduces to the 3-dB limit at zero temperature. Such a finding is compared, at fixed dynamical parameters, with the performance of homodyne continuous monitoring of the output modes, which allows one to beat coherent feedback and the 3-dB limit under certain dynamical conditions.
\end{abstract}

DOI: 10.1103/PhysRevResearch.2.043103

\section{INTRODUCTION}

Feedback is one of the main avenues to exert and refine control on physical systems. In quantum mechanics, feedback control may be applied in two, radically different, fashions: As measurement-based feedback [1], where measurements are used to purify the system and to inform operations on it, or as coherent feedback [2], where only deterministic manipulations of subsystems coupled to the system of interest are performed. While in measurement-based feedback the quantum information is turned into classical information by the act of measuring, in coherent feedback the information stays quantum at all stages of the control loop. It might be argued that, since the manipulations involved are deterministic, coherent feedback loops should not be considered as feedback control at all, but rather as a class of open-loop control strategies where only certain auxiliary degrees of freedom are accessible. However, quantum optics allows us to disregard such a terminological dispute (although the discussion of this issue in Ref. [3] is worth mentioning), through the adoption of the input-output formalism, which is tailored to describe the interaction of a countable set of localized modes (e.g., a set of cavity modes) with a neighboring field's continuum (the electromagnetic field outside a cavity). We shall, as customary in the context of quantum optics [4-6], define a coherent feedback loop as one where a set of output modes, interacting with a system at an input-output interface, may be manipulated through quantum CP maps and then fed back into a system as input modes at another interface. This approach is similar to that used in the established field of "cascaded quantum systems," where the output of one system is used as the input

Published by the American Physical Society under the terms of the Creative Commons Attribution 4.0 International license. Further distribution of this work must maintain attribution to the author(s) and the published article's title, journal citation, and DOI. of another [7-10], and of the related "all-optical" feedback [11], the difference being that here the output is fed back into the system from whence it came.

Notice that the input-output paradigm finds successful and broad application to a number of quantum systems where a high degree of coherent control is achievable, ranging from purely optical setups to optomechanics [12], nanoelectromechanics, atomic ensembles, and cavity QED waveguides [13], to mention but a few. Given the impressive recent advances in the realization of quantum technologies, connectivities (especially via fibers and waveguides) are quickly nearing a point where quantum control loops will be feasible and pivotal in harnessing quantum resources, such as quantum coherence and entanglement. Indeed, coherent control loops have been demonstrated in optical [5,14] and solid-state [15] systems, while measurement-based feedback has been by now applied to a variety of systems, with the aim of performing quantum operations, enhancing cooling routines [16], or entangling quantum systems [17]. It is therefore paramount to understand the ultimate limits of feedback strategies as well as which class of loops, coherent or measurement based, is advantageous to perform a given task or optimize a given figure of merit.

After the seminal study [11]—which unlike the present inquiry does not deal with a squeezing Hamiltonian acting on the system but rather with more general forms of coupling between system cavity and the feedback loop-such a theoretical comparison has been addressed only for finitedimensional scenarios $[3,18]$, for linear quadratic Gaussian optimization [4], or for out-of-loop figures of merit [6] (i.e., concerning the relationship between input and output degrees of freedom). In our study, the input-output formalism, compounded with a general description of passive linear operations, will yield a framework for a comparison between "interferometric" coherent feedback and measurement-based feedback at given connectivities and other technical and environmental parameters (such as detection efficiencies and temperature). Let us remark that, in practice, the generation 
and injection of squeezed light needed to go beyond purely passive, interferometric schemes, would be highly challenging, while high-efficiency homodyne detection, which we shall adopt for measurement-based protocols, is comparatively more straightforward [19].

Note that the set of operations encompassed by coherent and measurement-based feedback differ, since the stochastic dynamics originating from measurements cannot be reduced to deterministic operations, which is the essence of the so-called "measurement problem" of quantum mechanics. Nevertheless, coherent feedback has been proven superior in a number of tasks and contexts, so that our ability to demonstrate situations where measurement-based schemes do in principle prove superior is all the more striking and consequential. It should be stressed that we are considering here only figures of merit at steady state, rather than at any transient time. Measurement-based strategies, it turns out, prove particularly effective in stabilizing in-loop figures of merit (i.e., quantities pertaining to the localized modes).

In this paper, we shall consider a set of bosonic modes coupled to a white-noise continuum at finite temperature and focus on the optimization of steady-state squeezing. First, as proof of principle, we shall present a simple coherent feedback loop using a single feedback mode subject to losses, show that it can enhance the achievable squeezing up to a 3-dB limit [20], and contrast it with what is achievable through (feasible) homodyne measurements of the output field. Then, we will proceed to consider the most general harmonic coherent feedback setup, letting an arbitrary number of output modes at one interface undergo the most general deterministic Gaussian completely positive (CP)-map before being fed back into the system. We shall then restrict this framework to consider only operations not involving any source of squeezing. The Gaussian CP map in the feedback loop will be taken to be the most general open, passive optical transformation, corresponding in practice to leakage, beam splitters, and phase shifters, i.e., the most general "interferometric" scheme. This will allow us to prove analytically that the simple setup considered beforehand is indeed optimal, within interferometric coherent feedback, for the generation and stabilization of single-mode squeezing. Furthermore, we shall extend our findings to certain multimode systems and show that the bound holds for pure multimode steady states too. Notice that, not surprisingly, steady-state squeezing could be enhanced if squeezing was included in-loop, either by considering a nonpassive CP map or by using a squeezed input state as in Ref. [21]. Since these schemes would imply a separate source of squeezing, the very resource whose generation we are tasked with optimizing, we are disregarding them.

The paper is organized as follows: In Sec. II, we introduced the general formalism of bosonic continuous variables and of input-output interfaces, which will be used to describe coherent feedback; in Sec. III, we report known benchmarks on the steady-state squeezing that can be attained without control and with homodyne monitoring; Sec. IV contains the general formalism and all our main findings on the optimal squeezing achievable through interferometric coherent feedback for single-mode systems; Sec. V contains some considerations on the multimode extension of our optimality results; Sec. VI concludes the paper with a summary and future perspec- tives. The detailed technical arguments and derivations are deferred to the Appendixes: Appendix A recalls the calculation of the squeezing achievable via homodyne detection, Appendix B contains all the derivations concerning coherent feedback, and in Appendix C, we flesh out two technical arguments regarding the multimode extension of our study.

\section{PRELIMINARIES}

\section{A. Continuous variable systems}

A system of $n$ bosonic modes can be described as a vector of operators $\hat{\boldsymbol{r}}=\left(\hat{x}_{1}, \hat{p}_{1} \ldots \hat{x}_{n}, \hat{p}_{n}\right)^{\top}$. These obey canonical commutation relations (CCR) $\left[\hat{x}_{j}, \hat{p}_{k}\right]=i \delta_{j k} \hat{\mathbb{1}}$, where $\hbar=1$. The CCR for multiple modes can be described using the symmetrized version of the commutator [22]: $\left[\hat{\boldsymbol{r}}, \hat{\boldsymbol{r}}^{\top}\right]=\hat{\boldsymbol{r}} \hat{\boldsymbol{r}}^{\top}-$ $\left(\hat{\boldsymbol{r}} \hat{\boldsymbol{r}}^{\top}\right)^{\top}=i \Omega_{n}$ where $\Omega_{n}$ is a $2 n \times 2 n$ matrix known as the symplectic form, $\Omega_{n}=\bigoplus_{j=1}^{n} \Omega_{1}$, with $\Omega_{1, j k}=\delta_{j+1, k}-\delta_{j, k+1}$ for $j, k=1,2$. We will often omit the subscript from $\Omega$, letting the context specify the appropriate dimension. The second statistical moments of a state are defined as follows: $\sigma=$ $\operatorname{Tr}\left[\left\{(\hat{\boldsymbol{r}}-\overline{\boldsymbol{r}}),(\hat{\boldsymbol{r}}-\overline{\boldsymbol{r}})^{\top}\right\} \hat{\rho}\right]$, in terms of a real, symmetric covariance matrix $(\mathrm{CM}) \boldsymbol{\sigma}$.

The steady states we will focus on are Gaussian states, which may be defined as the ground and thermal states of quadratic Hamiltonians and are fully characterized by first and second statistical moments. We shall be concerned with the properties of the second moments only, and thus neglect the first moments in what follows. Unitary operations which map Gaussian states into Gaussian states are those generated by a quadratic Hamiltonians. The effect of such operations on the vector of operators is a symplectic transformation $\hat{\boldsymbol{r}} \rightarrow S \hat{\boldsymbol{r}}$ where $S$ is a $2 n \times 2 n$ real matrix which satisfies $S \Omega S^{\top}=\Omega$. The corresponding effect on the covariance matrix of the system is the transformation $\sigma \rightarrow S \sigma S^{\top}$. In this study, we will make use of so-called "passive" transformations, which do not add any energy to the system and therefore do not perform any squeezing. Passive transformations must satisfy the extra constraint that $S$ is orthogonal, i.e., $S S^{\top}=\mathbb{1}$.

\section{B. The input-output formalism}

The input-output formalism is a method for dealing with the evolution of systems coupled to a noisy environment, consisting of a continuum of modes (e.g., the free electromagnetic field) [23]. The interaction of the system with such an environment can be modeled as a series of instantaneous interactions with different modes at different times. These modes can be thought of as interacting instantaneously with the system and then being scattered away, never to interact again. The incoming mode which interacts with the system at time $t$ is known as the input mode and is called $\hat{x}_{\text {in }}(t)$, while the mode scattered at time $t$ is called $\hat{x}_{\text {out }}(t)$ and is known as the output mode. The input modes satisfy the continuous CCR: $\left[\hat{\boldsymbol{r}}_{\text {in }}(t), \hat{\boldsymbol{r}}_{\mathrm{in}}^{\top}\left(t^{\prime}\right)\right]=$ $i \Omega \delta\left(t-t^{\prime}\right)$, where $\hat{\boldsymbol{r}}_{\text {in }}(t)=\left(\hat{x}_{\mathrm{in}, 1}, \hat{p}_{\text {in }, 1} \ldots \hat{x}_{\mathrm{in}, m}, \hat{p}_{\text {in }, m}\right)^{\top}$. The most general coupling to the input fields is given by the Hamiltonian $\hat{H}_{C}=\hat{\boldsymbol{r}}^{\top} C \hat{\boldsymbol{r}}_{\text {in }}$, in terms of the $2 n \times 2 m$ coupling matrix $C$. The Heisenberg evolution of the system operators is given by a stochastic differential equation known as the 
quantum Langevin equation [22]:

$$
d \hat{\boldsymbol{r}}(t)=A \hat{\boldsymbol{r}}(t) d t+\Omega C \hat{\boldsymbol{r}}_{\mathrm{in}}(t) d t,
$$

where the drift matrix $A$ is given by $A=\Omega H_{S}+\frac{1}{2} \Omega C \Omega C^{\top}$, with the symmetric square matrix $H_{S}$ specifying the system Hamiltonian according to $\hat{H}_{S}=\frac{1}{2} \hat{\boldsymbol{r}}^{\top} H_{S} \hat{\boldsymbol{r}}$. The vector $\hat{\boldsymbol{r}}_{\text {in }}(t)$ is a quantum stochastic process known as a quantum Wiener process which, in analogy with the classical Wiener process, obeys the relations $\left[\hat{\boldsymbol{r}}_{\text {in }}(t), \hat{\boldsymbol{r}}_{\text {in }}^{\top}(t)\right](d t)^{2}=i \Omega d t$ and $\operatorname{Tr}\left[\left\{\hat{\boldsymbol{r}}_{\text {in }}(t), \hat{\boldsymbol{r}}_{\text {in }}^{\top}(t)\right\} \hat{\varrho}\right](d t)^{2}=\sigma_{\text {in }} d t$, where $\boldsymbol{\sigma}_{\text {in }}$ is the covariance matrix of the input modes. This relationship implies $\delta$ correlations between bath modes interacting at different times (the well-known white-noise condition) and hence the Markovianity of the free dynamics, which we are thus assuming.

The definition of $\sigma$ and Eq. (1) can be combined to obtain an equation for the evolution of the system covariance matrix, $\dot{\sigma}=A \sigma+\sigma A^{\top}+D$, where $D=\Omega C \sigma_{\text {in }} C^{\top} \Omega^{\top}$ is known as the diffusion matrix. Setting $\dot{\sigma}=0$ yields a Lyapunov equation for the steady-state (stationary) solution, which exists if and only if the matrix $A$ is "Hurwitz," i.e., if all its eigenvalues have negative real parts. If this condition is satisfied, then the steady-state solution reads

$$
\boldsymbol{\sigma}_{\infty}=\int_{0}^{\infty} e^{A t} D e^{A^{\top} t} d t .
$$

\section{BENCHMARKS}

\section{A. Squeezing with no control}

Our figure of merit to quantify squeezing is $\sigma_{11}$, the element of the covariance matrix corresponding to twice the variance of the $\hat{x}$ quadrature. As the value of $\sigma_{11}$ decreases, so does the uncertainty on $\hat{x}$, and the squeezing increases. As a typical case, let us consider here a single bosonic cavity mode, subject to the Hamiltonian $\hat{H}=\hat{H}_{S}+\hat{H}_{C}$, where $\hat{H}_{S}=-\frac{\chi}{4}\{\hat{x}, \hat{p}\}$ with $\chi>0$, corresponding to a Hamiltonian matrix $H_{S}=-\frac{\chi}{2} \sigma_{x}$ (where $\sigma_{x}$ is the Pauli $x$ matrix). Cavity losses are modeled by the external field coupling,

$$
\hat{H}_{C}=\sqrt{\gamma}\left(\hat{p} \hat{x}_{\text {in }}-\hat{x} \hat{p}_{\text {in }}\right),
$$

corresponding to a coupling matrix $C=\sqrt{\gamma} \Omega_{1}^{\top}$ where $\gamma$ is the strength of the coupling. When this is the form of systemenvironment coupling, the so-called input-output boundary condition relates the system modes to the input and output as follows [23]:

$$
\hat{\boldsymbol{r}}_{\text {out }}(t)=\sqrt{\gamma} \hat{\boldsymbol{r}}(t)-\hat{\boldsymbol{r}}_{\text {in }}(t) .
$$

Throughout this paper, we will consider input fields in Gibbs thermal states of their free Hamiltonian, so that $\sigma_{\text {in }}=N \mathbb{1}$, where $N=2 \bar{N}+1$ and $\bar{N} \geqslant 0$ is the mean number of thermal excitations in the environment, which can be related to temperature and frequency through the Bose law. The steady-state squeezing is $\sigma_{11}=N \gamma /(\chi+\gamma)$. The condition for stability is that $|\chi|<\gamma$, which means that, if the loss rate or squeezing parameter can be tuned, and the input fields are taken to be vacua (so $N=1$ ) the maximum steady-state squeezing that can be achieved is $\sigma_{11}=\frac{1}{2}$. This is known in the literature as the 3 -dB limit, as $10 \log _{10}(2) \approx 3.01$ (this is the noise, in decibels, associated with the smallest eigenvalue of $\sigma$ in units of vacuum noise).

\section{B. Homodyne monitoring}

Let us now modify the setting above by the continuous monitoring of the output $\hat{x}$ quadrature through a homodyne detector with efficiency $\zeta$, which yields a relevant CM's element given by

$$
\sigma_{11}^{m}=\frac{a+\sqrt{a^{2}+b}}{2 \zeta},
$$

for $a=\left[2 N \zeta-(1+(N-1) \zeta)\left(1+\frac{\chi}{\gamma}\right)\right]$ and $b=4 N \zeta(1-$ $\zeta$ ) (see Appendix A for a derivation of this). This monitoring maximizes the steady-state squeezing among all general-dyne detections at zero temperature, i.e., for $N=1$ [24], but is beneficial at finite temperature too (for $N>1$ ); we do not report the finite-temperature optimisation here, since it would require the unrealistic access to purifications of the bath [25]. Note that since the conditional CM (and hence the squeezing) does not depend on the measurement outcome, merely monitoring achieves the optimal feedback performance, without the need to perform any other control action.

\section{COHERENT FEEDBACK}

\section{A. "Simple" coherent feedback}

Let us begin our inquiry into coherent feedback by reporting on the performance of the simplest possible coherent feedback loop, obtained by feeding the output of one interface into the input of the other after undergoing losses [22]. We will therefore consider a system mode coupled to two input fields, each through a Hamiltonian of the form given in (3). To avoid ambiguity, we will use the subscript $e$ to refer to external, unmodified environmental modes. Coherent feedback involves setting $\hat{\boldsymbol{r}}_{\mathrm{in}, 1}=\hat{\boldsymbol{r}}_{e, 1}$ and $\hat{\boldsymbol{r}}_{\mathrm{in}, 2}(t)=\Phi\left(\hat{\boldsymbol{r}}_{\mathrm{out}, 1}(t)\right)$, where $\Phi$ is the CP map corresponding to losses. These losses can be modeled as mixing at a beam splitter with an environmental mode $\hat{\boldsymbol{r}}_{e, 2}$. This means that coherent feedback can be achieved by setting

$$
\begin{aligned}
\hat{\boldsymbol{r}}_{\mathrm{in}, 2} & =\sqrt{\eta} \hat{\boldsymbol{r}}_{\mathrm{out}, 1}+\sqrt{1-\eta} \hat{\boldsymbol{r}}_{e, 2} \\
& =\sqrt{\eta}\left(\sqrt{\gamma} \hat{\boldsymbol{r}}-\hat{\boldsymbol{r}}_{e, 1}\right)+\sqrt{1-\eta} \hat{\boldsymbol{r}}_{e, 2},
\end{aligned}
$$

where $\eta$ is the loss rate and we have used the input-output relation (B7). We are assuming instantaneous feedback, with no delays between the mode put out at interface 1 and fed back at interface 2, which will preserve the Markovianity of the dynamics. Making this substitution into Eq. (3) results in the system $\hat{\boldsymbol{r}}$ being effectively coupled to the environment $\hat{\boldsymbol{r}}_{e, \text { tot }}=\left(\hat{\boldsymbol{r}}_{e, 1}^{\top}, \hat{\boldsymbol{r}}_{e, 2}^{\top}\right)^{\top}$ through the coupling matrix $C=\sqrt{\gamma}(1-$ $\left.\sqrt{\eta} \Omega_{1}^{\top}, \sqrt{1-\eta} \Omega_{1}^{\top}\right)$. Such a system requires $\gamma(1-\sqrt{\eta})>\frac{\chi}{2}$ in order to be stable. The steady-state squeezing achieved in these conditions is $\sigma_{11}=\frac{N \gamma(1-\sqrt{\eta})}{\frac{\chi}{2}+\gamma(1-\sqrt{\eta})}$. This is minimized by letting $\sqrt{\eta} \rightarrow 1-\frac{\chi}{2 \gamma}$, which results in a squeezing of $\sigma_{11} \rightarrow \frac{N}{2}$. Thus, at zero temperature (i.e., for $N=1$ ), coherent feedback allows the 3-dB limit to be approached (but not beaten) for any choice of parameters satisfying $0<\frac{\chi}{2 \gamma}<1$. Notice that, regardless of the strength $\chi$, no stable squeezing is achievable if $N \geqslant 2$.

This is a very remarkable result, showing that a coherent feedback loop is in principle capable to amplify the 


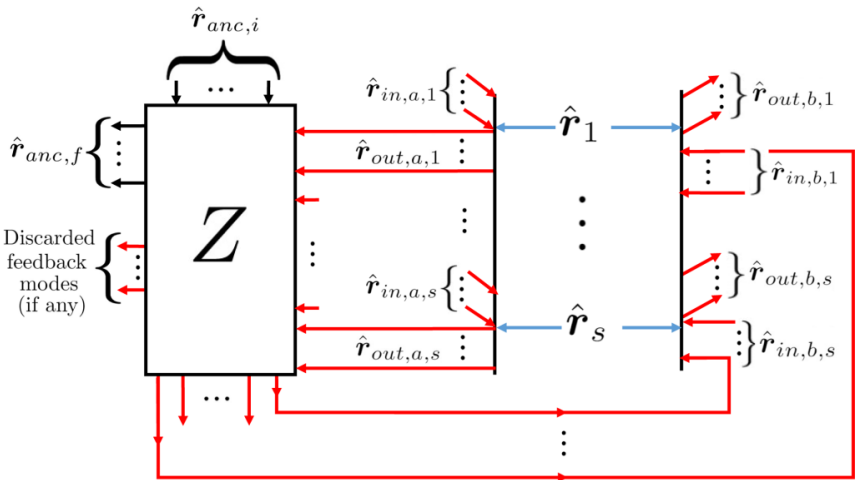

FIG. 1. The general coherent feedback scheme considered in this paper. System modes are labeled in blue, the modes involved in the coherent feedback loop are red, and ancilla modes are black. A transformation $Z$ is made jointly on the feedback and ancilla modes, before some are traced out and the remaining modes are fed back into the cavity. Our framework allows for the possibility of some feedback modes being discarded before the rest are fed back into the system.

strength of any squeezing Hamiltonian up to the 3-dB stability limit. However, a homodyne measurement-based loop, whose steady-state squeezing is given by Eq. (5), outperforms this coherent feedback scheme when the efficiency $\zeta$ of the detector satisfies

$$
\zeta \geqslant \frac{2(\gamma-\chi)}{2(\gamma-\chi)+N(2 \chi-\gamma)}
$$

and the denominator of the right-hand side (RHS) of (7) is positive [26]. For $\chi<\gamma / 2$, either the denominator is negative or the bound above is larger than 1, which proves that homodyne monitoring does not beat coherent feedback at such weak interaction strengths. For $\chi \geqslant \gamma / 2$, there is always a detection efficiency threshold above which the coherent feedback loop we considered is outperformed by homodyne monitoring; this threshold, quite interestingly, decreases with increasing noise (although the absolute performance of monitoring at given $\chi$ still deteriorates as the noise increases). As the upper limit for stability $\chi=\gamma$ is approached, the efficiency threshold falls to zero, so that detection with any efficiency will be better than our coherent loop in this limit. The ultimate performance of homodyne monitoring is obtained at $\zeta=1$, where $\sigma_{11}^{m}=N(1-\chi / \gamma)$ : Hence, monitoring can in principle achieve stable squeezing $\left(\sigma_{11}^{m}<1\right)$ for all values of $N$, although only for $\chi>\gamma(1-1 / N)$. Notice that, in principle, arbitrarily high squeezing may be stabilized at all noises (temperatures), whereas the coherent feedback loop we studied is bounded by the value $N / 2$. In order to achieve a conclusive comparison between coherent and measurement-based loops, we need to extend our treatment beyond a specific coherent feedback loop to include any possible interferometric scheme without additional sources of squeezing.

\section{B. General coherent feedback}

Let us therefore consider the most general coherent feedback protocol, as depicted in Fig. 1. A system consisting of $s$ bosonic modes is coupled to $p+q$ input modes through a coupling Hamiltonian of the same form as (3). Each system mode $\hat{\boldsymbol{r}}_{j}$ is coupled to $l_{j}+m_{j}$ input-output interfaces with $l_{j}$, $m_{j}$ such that $p=\sum_{j=1}^{s} l_{j}$ and $q=\sum_{j=1}^{s} m_{j}$. We will give the label $a$ to the modes interacting at the first $p$ input-output interfaces, and the label $b$ to the modes interacting at the remaining $q$ interfaces. The first $p$ input modes are environmental white noise, meaning we can write $\hat{\boldsymbol{r}}_{\mathrm{in}, a}=\hat{\boldsymbol{r}}_{e, a}=\left(\hat{\boldsymbol{r}}_{e, 1}^{\top} \ldots \hat{\boldsymbol{r}}_{e, p}^{\top}\right)^{\top}$. The corresponding output modes then undergo the most general Gaussian CP map. This is achieved by applying a symplectic transformation $Z$ on the output modes, along with $n$ ancillary white noise modes before tracing out the ancillas. Later, we restrict ourselves to the case where this CP map is passive and therefore includes no extra source of squeezing, (a scenario which we refer to as "interferometric," since it can be reproduced entirely with passive optics). After the transformation, the resulting $q$ modes are fed into the system through the remaining $q$ input interfaces. We shall assume that the additional ancillary modes are also affected by the same thermal noise as the environment, so that it is still $\sigma_{\text {in }}=N \mathbb{1}$ [27].

The transformation on the output and ancilla modes can be represented as an symplectic, $2(p+n)$-dimensional, square matrix $Z$ :

$$
\hat{\boldsymbol{r}}_{\mathrm{in}, b} \oplus \hat{\boldsymbol{r}}_{a n c, f}=Z\left(\hat{\boldsymbol{r}}_{\mathrm{out}, a} \oplus \hat{\boldsymbol{r}}_{a n c, i}\right),
$$

where $\quad \hat{\boldsymbol{r}}_{\mathrm{in}, b}=\left(\hat{\boldsymbol{r}}_{\mathrm{in},(p+1)}^{\top} \ldots \hat{\boldsymbol{r}}_{\mathrm{in},(p+q)}^{\top}\right)^{\top} \quad$ and $\quad \hat{\boldsymbol{r}}_{\mathrm{out}, a}=$ $\left(\hat{\boldsymbol{r}}_{\text {out }, 1}^{\top} \ldots \hat{\boldsymbol{r}}_{\text {out }, p}^{\top}\right)^{\top}$. Later, by requiring that this matrix is also orthogonal, we ensure that it corresponds to a passive transformation involving no extra squeezing. The initial and final states of the ancilla modes are given by $\hat{\boldsymbol{r}}_{a n c, i}$ and $\hat{\boldsymbol{r}}_{a n c, f}$ respectively where $\hat{\boldsymbol{r}}_{a n c, i}=\left(\hat{\boldsymbol{r}}_{e,(p+1)}^{\top} \ldots \hat{\boldsymbol{r}}_{e,(p+n)}^{\top}\right)^{\top}$. The symplectic matrix $Z$ can be decomposed into block matrices $Z=\left(\begin{array}{ll}E & F \\ G & H\end{array}\right)$. This representation of $Z$ allows us to write $\hat{\boldsymbol{r}}_{\mathrm{in}, b}=E \hat{\boldsymbol{r}}_{\mathrm{out}, a}+F \hat{\boldsymbol{r}}_{a n c, i}$. It is shown in Appendix B that the overall effect of this coherent feedback protocol is to couple the system modes to the white-noise environment, now given by $\hat{\boldsymbol{r}}_{e, a} \oplus \hat{\boldsymbol{r}}_{\text {anc }}$, through the coupling matrix $C_{c f}=\left(K_{p}-K_{q} E \quad \mid K_{q} F\right)$, where $K_{p}=\bigoplus_{j=1}^{s} C_{l_{j}}$, and $C_{j}$ is the $2 \times 2 j$-dimensional matrix of the form $\sqrt{\gamma}\left(\Omega^{\top} \ldots \Omega^{\top}\right)$. The matrix $K_{q}$ is of the same form as $K_{p}$, but with dimension $2 s \times 2 q$. It is also shown that adding coherent feedback modifies the system Hamiltonian matrix $H_{S}$ by addition of the matrix $H_{c f}=H_{S}+K_{q} E B+B^{\top} E^{\top} K_{q}^{\top}$, where $B=\bigoplus_{j=1}^{n} \Gamma_{l_{j}}$, and $\Gamma_{l}=\sqrt{\gamma}\left(\mathbb{1}_{2} \ldots \mathbb{1}_{2}\right)^{\top}$ is a $2 l \times 2$ matrix.

\section{Optimal interferometric feedback}

An elegant and compact description of all interferometric schemes mediating the coherent feedback loop is achieved by requiring that the matrix $Z$ is orthogonal as well as symplectic, amounting to $E E^{\top}+F F^{\top}=\mathbb{1}$. As shown in Appendix B 3, after this restriction is made the drift matrix takes the form

$$
A_{c f}=\Omega H_{c f}-\frac{1}{2} D_{0},
$$

where $D_{0}$ is the diffusion matrix of the system under the same setup at zero temperature, i.e., $D_{0}=D / N$.

Let us now now determine the optimal single-mode steadystate squeezing achievable through interferometric coherent feedback and show that the $3-\mathrm{dB}$ squeezing limit cannot be 
beaten, even allowing for arbitrary control on the quadratic system Hamiltonian.

The steady-state $\mathrm{CM}, \sigma_{\infty}$, solves the Lyapunov equation $\sigma_{\infty} A^{\top}+A \sigma_{\infty}+D=0$. For a single mode, the squeezing is given by the CM's smallest eigenvalue, which we will label $\sigma_{1}$, with a corresponding eigenvector $\boldsymbol{v}$. The Lyapunov equation implies: $\sigma_{1}=\frac{-v^{\dagger} D v}{v^{\dagger}\left(A+A^{\top}\right) v}$. After substituting Eq. (9) for $A$ and $D=N D_{0}$, we obtain

$$
\sigma_{1}=\frac{N \boldsymbol{v}^{\dagger} D_{0} \boldsymbol{v}}{\boldsymbol{v}^{\dagger} D_{0} \boldsymbol{v}-\boldsymbol{v}^{\dagger}\left(\Omega H_{c f}+H_{c f} \Omega^{\top}\right) \boldsymbol{v}}=\frac{N \delta}{\delta-\alpha},
$$

where $\delta=\boldsymbol{v}^{\dagger} D_{0} \boldsymbol{v}>0$ and $\alpha=\boldsymbol{v}^{\dagger}\left(\Omega H_{c f}+H_{c f} \Omega\right) \boldsymbol{v}$. Now, the vector $\boldsymbol{w}=\Omega \boldsymbol{v}$ is orthogonal to $\boldsymbol{v}$, due to the skew symmetry of $\Omega$. Since $\sigma_{\infty}$ is a $2 \times 2$ symmetric matrix, $\boldsymbol{w}$ must also be an eigenvector of $\sigma_{\infty}$ with eigenvalue $\sigma_{2}$.

In Appendix B, it is shown that $D_{0}$ satisfies $\Omega^{\top} D_{0} \Omega=D_{0}$. It can also be seen that $\Omega^{\top}\left(\Omega H_{c f}+H_{c f} \Omega^{\top}\right) \Omega=-\left(\Omega H_{c f}+\right.$ $H_{c f} \Omega^{\top}$ ), because $\Omega$ is skew symmetric and orthogonal. Hence, $\boldsymbol{w}^{\dagger} D_{0} \boldsymbol{w}=\boldsymbol{v}^{\dagger} D_{0} \boldsymbol{v}=\delta$ and $\boldsymbol{w}^{\dagger}\left(\Omega H_{c}+H_{c} \Omega^{\top}\right) \boldsymbol{w}=$ $-\boldsymbol{v}^{\dagger}\left(\Omega H_{c}+H_{c} \Omega^{\top}\right) \boldsymbol{v}=-\alpha$. The second eigenvalue of $\sigma_{\infty}$ can therefore be found by multiplying the Lyapunov equation from the left and right by $\boldsymbol{w}$ and rearranging, yielding $\sigma_{2}=$ $\frac{N \boldsymbol{w}^{\dagger} D_{0} \boldsymbol{w}}{\boldsymbol{w}^{\dagger} D_{0} \boldsymbol{w}-\boldsymbol{w}^{\dagger}\left(\Omega H_{c f}+H_{c f} \Omega\right) \boldsymbol{w}}=\frac{N \delta}{\delta+\alpha}$. But then, $\sigma_{2}>0$ implies $\alpha>$ $-\delta$, which can be inserted into Eq. (10) to get $\sigma_{1}=\frac{N \delta}{\delta-\alpha}>\frac{N}{2}$. We therefore conclude that interferometric coherent feedback cannot beat the 3-dB limit and that the simple setup described earlier is indeed optimal. Note that this bound was derived for a fully general (quadratic) system Hamiltonian.

\section{THE MULTIMODE CASE}

Our general framework extends to multiple system modes. However, the proof above does not, and it can in fact be shown (see Appendix C) that the smallest eigenvalue of a multimode $\sigma_{\infty}$ can be decreased below 0.5 . Yet, several interesting remarks can be made. For many modes, the smallest eigenvalue of $\sigma_{\infty}$ does not quantify the squeezing any longer, since it will not in general represent the noise of a physical quadrature. This is the case only if $\sigma_{\infty}$ may be diagonalized by an orthogonal symplectic transformation: Under such a condition, the 3-dB bound still applies, as proven in Appendix B. Notably, this is the case of globally pure Gaussian states (whose CM may always be diagonalized by a passive symplectic [22]), so a coherent feedback scheme that also purifies the global state cannot beat the 3-dB bound. Moreover, since all single-mode CMs may be diagonalized by passive symplectic transformations (i.e., $2 \times 2$ rotations), no scheme that would purify locally the degree of freedom attaining maximum squeezing can beat the 3-dB bound (more generally, this extends to all schemes that would render such a mode uncorrelated with all other system modes). Numerical evidence suggests the 3-dB bound does hold for all diagonal elements attainable from a multimode $\sigma_{\infty}$ through orthogonal symplectic transformations (that is, for the noise of any physical quadrature), although we do not possess conclusive proof in this regard, so we leave it as a conjecture.

\section{CONCLUSIONS AND SUMMARY}

To summarize, we have developed a general treatment of interferometric coherent feedback in the Gaussian regime and shown that no protocol within this framework can beat the 3-dB squeezing limit at steady state. In contrast, homodyne monitoring of output fields can stabilize arbitrarily high squeezing at sufficiently low noise provided that detection efficiency is high enough.

Specifically, in regard to the squeezing Hamiltonian $-\frac{\chi}{4}\{\hat{x}, \hat{p}\}$, coherent feedback loops are superior for $\chi<\gamma / 2$ (and, quite remarkably, attain optimal performances independent from the interaction strength), while measurement-based, homodyne feedback is better for $\chi \geqslant \gamma / 2$ and efficiencies satisfying (7). Our comparison is definitive at zero temperature, for $N=1$, in the sense that both measurement-based and coherent feedback were fully optimized for vacuum input noise (homodyning is then optimal), and that at optical frequencies one has $(N-1) \approx 10^{-6}$. Our findings hinge on the phase-insensitive nature of the input-output coupling (3), which implies a diffusion matrix $D$ proportional to the identity, and would not apply, for instance, to quantum Brownian motion. In this sense, our results may be considered as an extension of the well-known 3-dB squeezing limit that affects phase-insensitive amplifiers [20]. Beside the phase insensitivity of the coupling (3), stability is the other essential ingredient to establish the bound as, it should be noted, unstable coherent feedback loops would be able to achieve higher squeezing (but are typically not desirable in practice).

This study lays the groundwork for further inquiries on interferometric coherent feedback, which may be extended to the optimisation of entanglement, to more general noise models, and to the cooling of concrete systems, such as quantum optomechanics [28-30].

\section{ACKNOWLEDGMENTS}

We acknowledge discussions with M. Brunelli, who made us aware of additional literature, and M. Genoni, who pointed out inaccuracies.

\section{APPENDIX A: HOMODYNE MONITORING AT FINITE TEMPERATURE}

Continuous, general-dyne monitoring of the output field turns the diffusive equation $\dot{\sigma}=A \sigma+\sigma A^{\top}+D$ into the following Riccati equation (see, e.g., Ref. [22] for a complete treatment of the theory):

$$
\dot{\sigma}=\tilde{A} \sigma+\sigma \tilde{A}^{\top}+\tilde{D}-\sigma B B^{\top} \sigma,
$$

for

$$
\begin{gathered}
\tilde{A}=A+G B^{\top}, \quad \tilde{D}=D-G G^{\top}, \\
B=C \Omega\left(\sigma_{\text {in }}+\sigma_{m}\right)^{-1 / 2} \quad G=\Omega C \sigma_{\text {in }}\left(\sigma_{\text {in }}+\sigma_{m}\right)^{-1 / 2},
\end{gathered}
$$

where the covariance matrix $\sigma_{m}$ parametrizes the choice of measurement. We will consider the homodyne detection of the output field of a single-input, single-output system as described in the section titled "Squeezing with no control." 
Homodyne detection of the $\hat{x}$ quadrature with efficiency $\zeta$ is obtained by setting

$$
\sigma_{m}=\lim _{z \rightarrow 0}\left(\begin{array}{cc}
\frac{z+1-\zeta}{\zeta} & 0 \\
0 & \frac{\frac{1}{z}+1-\zeta}{\zeta}
\end{array}\right),
$$

which leads to a diagonal quadratic equation for the monitored steady-state covariance matrix, whose diagonal elements $\sigma_{11}^{m}$ and $\sigma_{22}^{m}$ must satisfy (the off-diagonal elements vanish)

$$
\begin{gathered}
\frac{\gamma \zeta}{\zeta(N-1)+1} \sigma_{11}^{m 2}+\left[\gamma+\chi-2 \frac{\gamma \zeta N}{\zeta(N-1)+1}\right] \sigma_{11}^{m}+\frac{\gamma \zeta N^{2}}{\zeta(N-1)+1}-\gamma N=0, \\
(\gamma-\chi) \sigma_{22}^{m}-\gamma N=0
\end{gathered}
$$

with physical solutions

$$
\begin{gathered}
\sigma_{11}^{m}=\frac{2 N \zeta-[1+(N-1) \zeta]\left(1+\frac{\chi}{\gamma}\right)+\sqrt{\left\{2 N \zeta-[1+(N-1) \zeta]\left(1+\frac{\chi}{\gamma}\right)\right\}^{2}+4 N \zeta(1-\zeta)}}{2 \zeta}, \\
\sigma_{22}^{m}=\frac{N}{1-\frac{\chi}{\gamma}} .
\end{gathered}
$$

The other solution for $\sigma_{11}^{m}$ must be discarded since it is negative or zero and would thus violate the strict positivity of $\boldsymbol{\sigma}^{m}$, stemming from the uncertainty principle. The solution for $\sigma_{22}^{m}$ shows that, even under monitoring, the condition $|\chi|<\gamma$ is necessary for stability.

\section{APPENDIX B: A GENERAL FRAMEWORK FOR GAUSSIAN COHERENT FEEDBACK}

We will consider $s$ bosonic system modes interacting with an environment hosting a coherent feedback loop according to the general scheme of Fig. 1. Each mode will be coupled to an arbitrary number of environmental white noise modes through an exchange of excitations Hamiltonian. The $j$ th system mode will be coupled to $l_{j}+m_{j}$ input-out interfaces. The outputs of the first $l_{j}$ interfaces for each mode will be used to create a coherent feedback loop. We will label the total number of output modes used in coherent feedback as $p=\sum_{j} l_{j}$. The number of interfaces whose outputs are not used for coherent feedback is given by $q=\sum_{j} m_{j}$. The $p$ output modes are then collectively subjected to the most general Gaussian CP map. After this, they will then be fed back into the remaining $q$ input-output interfaces.

When no coherent feedback is present, all of the $p+q$ interfaces couple the system to a white-noise environment. The coupling Hamiltonian matrix $\hat{H}_{C}$ for this interaction can be described in terms of a coupling Hamiltonian $H$ which satisfies $\hat{H}_{C}=\frac{1}{2} \hat{\boldsymbol{r}}_{\text {tot }}^{\mathrm{T}} H \hat{\boldsymbol{r}}_{\text {tot }}$. The vector $\hat{\boldsymbol{r}}_{\text {tot }}$ is the total vector of all system and input modes $\hat{\boldsymbol{r}}_{\text {tot }}=\hat{\boldsymbol{r}} \oplus \hat{\boldsymbol{r}}_{\mathrm{in}, \text { tot }}$ where $\hat{\boldsymbol{r}}=\hat{\boldsymbol{r}}_{1} \oplus$ $\hat{\boldsymbol{r}}_{2} \cdots \oplus \hat{\boldsymbol{r}}_{s}$ is the vector of system modes and $\hat{\boldsymbol{r}}_{j}=\left(\hat{x}_{j}, \hat{p}_{j}\right)^{\mathrm{\top}}$. Similarly, the total vector of all input modes is given by $\hat{\boldsymbol{r}}_{\mathrm{in}, \mathrm{tot}}=\hat{\boldsymbol{r}}_{\mathrm{in}, 1} \oplus \hat{\boldsymbol{r}}_{\mathrm{in}, 2} \cdots \oplus \hat{\boldsymbol{r}}_{\mathrm{in}, p+q}$. The Hamiltonian coupling matrix $H_{C}$ is given by

$$
H_{C}=\left(\begin{array}{ccc}
0_{s \times s} & K_{l} & K_{m} \\
K_{l}^{\top} & 0 & 0 \\
K_{m}^{\top} & 0 & 0
\end{array}\right),
$$

with

$$
K_{l}=\left(\begin{array}{ccccc}
C_{l_{1}} & 0 & 0 & \ldots & 0 \\
0 & C_{l_{2}} & 0 & \ldots & 0 \\
\vdots & & \ddots & & \vdots \\
0 & \ldots & 0 & \ldots & C_{l_{s}}
\end{array}\right)
$$

and

$$
K_{m}=\left(\begin{array}{ccccc}
C_{m_{1}} & 0 & 0 & \ldots & 0 \\
0 & C_{m_{2}} & 0 & \ldots & 0 \\
\vdots & & \ddots & & \vdots \\
0 & \ldots & 0 & \ldots & C_{m_{s}}
\end{array}\right)
$$

where $C_{j}$ indicates a $2 \times 2 j$ matrix of the form $\sqrt{\gamma}\left(\Omega^{\top} \ldots \Omega^{\top}\right)$.

We will use the label $a$ to indicate the set of interfaces whose outputs are used for coherent feedback, so $\hat{\boldsymbol{r}}_{\text {out }, a, 1}$ is a $2 l_{1}$-dimensional vector of output modes corresponding to system mode 1 . The total output at $a$ will be labeled by the $2 p$ dimensional vector $\hat{\boldsymbol{r}}_{\text {out }, a}=\hat{\boldsymbol{r}}_{\text {out }, a, 1} \oplus \hat{\boldsymbol{r}}_{\text {out }, a, 2} \oplus \cdots \oplus \hat{\boldsymbol{r}}_{\text {out }, a, s}$.

These output modes can be related to the system and environmental white-noise modes through an input-output relation. For a single-system mode coupled to multiple inputoutput interfaces, this relation takes the form

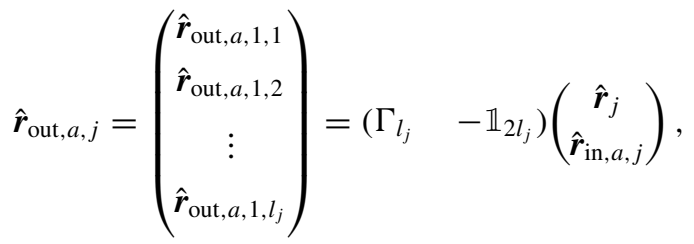

where $\Gamma_{k}$ indicates a $2 k \times 2$ matrix of the form $\sqrt{\gamma}\left(\mathbb{1}_{2} \ldots \mathbb{1}_{2}\right)^{\top}$ and $\hat{\boldsymbol{r}}_{\mathrm{in}, a, j}$ is a $2 l_{j}$-dimensional vector of input modes $\hat{\boldsymbol{r}}_{\mathrm{in}, a, j}=\left(\hat{\boldsymbol{r}}_{\mathrm{in}, a, j, 1} \oplus \cdots \oplus \hat{\boldsymbol{r}}_{\mathrm{in}, a, j, l_{j}}\right)$. 
The multimode version of the input output relation therefore reads

$$
\hat{\boldsymbol{r}}_{\mathrm{out}, a}=\left(\begin{array}{c}
\hat{\boldsymbol{r}}_{\mathrm{out}, a, 1} \\
\vdots \\
\hat{\boldsymbol{r}}_{\mathrm{out}, a, s}
\end{array}\right)=\left(\begin{array}{ccccccccc}
\Gamma_{l_{1}} & 0 & \ldots & 0 & -\mathbb{1}_{2 l_{1}} & 0 & \ldots & \ldots & 0 \\
0 & \Gamma_{l_{2}} & 0 & \ldots & 0 & -\mathbb{1}_{2 l_{2}} & 0 & \ldots & 0 \\
0 & 0 & \ddots & 0 & \ldots & 0 & \ddots & \ldots & 0 \\
0 & \ldots & 0 & \Gamma_{l_{s}} & 0 & \ldots & 0 & \ldots & -\mathbb{1}_{2 l_{s}}
\end{array}\right)\left(\begin{array}{c}
\hat{\boldsymbol{r}}_{1} \\
\hat{\boldsymbol{r}}_{2} \\
\vdots \\
\hat{\boldsymbol{r}}_{s} \\
\hat{\boldsymbol{r}}_{\mathrm{in}, a, 1} \\
\hat{\boldsymbol{r}}_{\mathrm{in}, a, 2} \\
\vdots \\
\hat{\boldsymbol{r}}_{\mathrm{in}, a, s}
\end{array}\right) .
$$

We will define the matrix $B$ as

$$
B=\left(\begin{array}{cccc}
\Gamma_{l_{1}} & 0 & \ldots & 0 \\
0 & \Gamma_{l_{2}} & \ldots & 0 \\
\vdots & \vdots & \ddots & 0 \\
0 & 0 & 0 & \Gamma_{l_{s}}
\end{array}\right),
$$

which allows us to write the multimode input-output relation as

$$
\hat{\boldsymbol{r}}_{\mathrm{out}, a}=\left(\begin{array}{ll}
B & -\mathbb{1}_{2 p}
\end{array}\right)\left(\begin{array}{c}
\hat{\boldsymbol{r}} \\
\hat{\boldsymbol{r}}_{\mathrm{in}, a}
\end{array}\right) .
$$

We will now consider the action of a Gaussian CP map on these output modes. This involves a symplectic operation on the joint state of the output modes and an arbitrary number of ancilla modes, which are then traced out. The symplectic matrix corresponding to this transformation will be called $Z$ and we will write it in terms of its block matrices:

$$
Z=\left(\begin{array}{ll}
E & F \\
G & H
\end{array}\right) .
$$

The action of $Z$ on the output and ancilla modes is

$$
\begin{aligned}
Z\left(\hat{\boldsymbol{r}}_{\text {out }, a} \oplus \hat{\boldsymbol{r}}_{\text {anc }}\right) & =\left(\begin{array}{ll}
E & F \\
G & H
\end{array}\right)\left(\begin{array}{l}
\hat{\boldsymbol{r}}_{\text {out }, a} \\
\hat{\boldsymbol{r}}_{\text {anc }, i}
\end{array}\right) \\
& =\left(\begin{array}{c}
E \hat{\boldsymbol{r}}_{\text {out }, a}+F \hat{\boldsymbol{r}}_{\text {anc }, i} \\
G \hat{\boldsymbol{r}}_{\text {out }, a}+F \hat{\boldsymbol{r}}_{\text {anc }, i}
\end{array}\right),
\end{aligned}
$$

where $\hat{\boldsymbol{r}}_{a n c, i}$ indicates a $2 n$-dimensional vector representing the initial state of $n$ ancilla modes, which are taken to be white-noise environmental modes. After tracing out the ancilla modes, this corresponds to a CP map of the which sends $\hat{\boldsymbol{r}}_{\text {out }, a} \mapsto E \hat{\boldsymbol{r}}_{\text {out }, a}+F \hat{\boldsymbol{r}}_{\text {anc }, i}$. We note that later on, we will assume that the operation performed in loop is passive, so it adds no extra squeezing. This means that $Z$ is orthogonal so $Z Z^{\top}=\mathbb{1}$. However, the framework developed in this section makes no such assumption and is therefore completely general in the Gaussian regime.

To avoid ambiguity, from now on we will use a subscript $e$ to indicate white noise modes and a subscript in to indicate the input modes interacting through at the input-output interface. We will label the remaining $q$ input-ouput interfaces (whose outputs are not used for coherent feedback) with $b$. Adding coherent feedback involves setting $\hat{\boldsymbol{r}}_{\mathrm{in}, b}=E \hat{\boldsymbol{r}}_{\text {out }, a}+F \hat{\boldsymbol{r}}_{\text {anc }}$ and $\hat{\boldsymbol{r}}_{\mathrm{in}, a}=\hat{\boldsymbol{r}}_{e, b}$. Writing this in matrix form gives

$$
\left(\begin{array}{c}
\hat{\boldsymbol{r}} \\
\hat{\boldsymbol{r}}_{\mathrm{in}, a} \\
\hat{\boldsymbol{r}}_{\mathrm{in}, b}
\end{array}\right)=\left(\begin{array}{cccc}
\mathbb{1}_{2 s} & 0 & 0 & 0 \\
0 & \mathbb{1}_{2 p} & 0 & 0 \\
0 & 0 & E & F
\end{array}\right)\left(\begin{array}{c}
\hat{\boldsymbol{r}} \\
\hat{\boldsymbol{r}}_{e, a} \\
\hat{\boldsymbol{r}}_{\mathrm{out}, a} \\
\hat{\boldsymbol{r}}_{\text {anc }}
\end{array}\right) .
$$

We can also use the multimode input-output relation (B7) to write

$$
\left(\begin{array}{c}
\hat{\boldsymbol{r}} \\
\hat{\boldsymbol{r}}_{e, a} \\
\hat{\boldsymbol{r}}_{\mathrm{out}, a} \\
\hat{\boldsymbol{r}}_{\text {anc }}
\end{array}\right)=\left(\begin{array}{ccc}
\mathbb{1}_{2 s} & 0 & 0 \\
0 & \mathbb{1}_{2 p} & 0 \\
B & -\mathbb{1}_{2 p} & 0 \\
0 & 0 & \mathbb{1}_{2 n}
\end{array}\right)\left(\begin{array}{c}
\hat{\boldsymbol{r}} \\
\hat{\boldsymbol{r}}_{e, a} \\
\hat{\boldsymbol{r}}_{\text {anc }}
\end{array}\right) .
$$

Combining these two equations, we can write

$$
\begin{aligned}
\left(\begin{array}{c}
\hat{\boldsymbol{r}} \\
\hat{\boldsymbol{r}}_{\text {in }, a} \\
\hat{\boldsymbol{r}}_{\text {in }, b}
\end{array}\right) & =\left(\begin{array}{cccc}
\mathbb{1}_{2 s} & 0 & 0 & 0 \\
0 & \mathbb{1}_{2 p} & 0 & 0 \\
0 & 0 & E & F
\end{array}\right)\left(\begin{array}{ccc}
\mathbb{1}_{2 s} & 0 & 0 \\
0 & \mathbb{1}_{2 p} & 0 \\
B & -\mathbb{1}_{2 p} & 0 \\
0 & 0 & \mathbb{1}_{2 n}
\end{array}\right) \\
\left(\begin{array}{c}
\hat{\boldsymbol{r}} \\
\hat{\boldsymbol{r}}_{e, a} \\
\hat{\boldsymbol{r}}_{\text {anc }}
\end{array}\right) & =R\left(\begin{array}{c}
\hat{\boldsymbol{r}} \\
\hat{\boldsymbol{r}}_{e, a} \\
\hat{\boldsymbol{r}}_{\text {anc }}
\end{array}\right) .
\end{aligned}
$$

The effect of adding coherent feedback is therefore to couple the system to a white-noise environment given by $\left(\hat{\boldsymbol{r}}_{e, a}^{\top}, \hat{\boldsymbol{r}}_{a n c}^{\top}\right)^{\top}$ through a coupling Hamiltonian characterized by the matrix $H_{C}^{c f}=R^{\top} H_{C} R$. This matrix is

$$
H_{C}^{c f}=\left(\begin{array}{ccc}
K_{m} E B+B^{\top} E^{\top} K_{m}^{\top} & K_{l}-K_{m} E & K_{m} F \\
K_{l}^{\top}-E^{\top} K_{m}^{\top} & 0 & 0 \\
F^{\top} K_{m}^{\top} & 0 & 0
\end{array}\right),
$$

which couples the system to the environment through the Hamiltonian operator

$$
\hat{H}_{C}^{c f}=\frac{1}{2}\left(\hat{\boldsymbol{r}}^{\top}, \hat{\boldsymbol{r}}_{e, a}^{\top}, \hat{\boldsymbol{r}}_{a n c}^{\top}\right) H_{C}^{c f}\left(\hat{\boldsymbol{r}}^{\top}, \hat{\boldsymbol{r}}_{e, a}^{\top}, \hat{\boldsymbol{r}}_{a n c}^{\top}\right)^{\top} .
$$

Notice that this results in a matrix equal to $K_{m} E B+B^{\top} E^{\top} K_{m}^{\top}$ being added to the system Hamiltonian matrix and changes the effective coupling matrix to

$$
C_{c f}=\left(K_{l}-K_{m} E \quad \mid \quad K_{m} F\right) .
$$


These changes, along with a system Hamiltonian and environmental input state, fully characterize the evolution of the system under coherent feedback.

\section{Properties of the orthogonal symplectic matrix}

We have considered a symplectic matrix of the form

$$
Z=\left(\begin{array}{ll}
E & F \\
G & H
\end{array}\right),
$$

which transformed a vector of operators as per $\hat{\boldsymbol{r}} \mapsto Z \hat{\boldsymbol{r}}$. Here, $E$ is a $(2 m \times 2 l)$ matrix and $F$ is a $(2 m \times 2 n)$ matrix. We will now restrict $Z$ to be an orthogonal symplectic matrix, which means that the operation it corresponds to is passive and therefore does not perform any squeezing. The condition of orthogonality means that $Z Z^{\top}=\mathbb{1}$, which gives us the following conditions on the submatrices:

$$
\begin{aligned}
Z Z^{\top} & =\left(\begin{array}{ll}
E & F \\
G & H
\end{array}\right)\left(\begin{array}{ll}
E^{\top} & G^{\top} \\
F^{\top} & H^{\top}
\end{array}\right) \\
& =\left(\begin{array}{ll}
E E^{\top}+F F^{\top} & E G^{\top}+F H^{\top} \\
G E^{\top}+H F^{\top} & G G^{\top}+H H^{\top}
\end{array}\right)=\left(\begin{array}{ll}
\mathbb{1} & 0 \\
0 & \mathbb{1}
\end{array}\right) .
\end{aligned}
$$

In particular, we shall make use of the relation $E E^{\top}+F F^{\top}=$ $\mathbb{1}$. The condition of symplecticity means that $Z \Omega Z^{\top}=\Omega$. Recall that we are using the convention that the dimension of $\Omega$ is specified by the context. In terms of the submatrices, this means that

$$
\begin{aligned}
Z \Omega Z^{\top} & =\left(\begin{array}{ll}
E & F \\
G & H
\end{array}\right)\left(\begin{array}{cc}
\Omega & 0 \\
0 & \Omega
\end{array}\right)\left(\begin{array}{cc}
E^{\top} & G^{\top} \\
F^{\top} & H^{\top}
\end{array}\right) \\
& =\left(\begin{array}{cc}
E \Omega E^{\top}+F \Omega F^{\top} & E \Omega G^{\top}+F \Omega H^{\top} \\
G \Omega E^{\top}+H \Omega F^{\top} & G \Omega G^{\top}+H \Omega H^{\top}
\end{array}\right) \\
& =\left(\begin{array}{ll}
\Omega & 0 \\
0 & \Omega
\end{array}\right) .
\end{aligned}
$$

From this, we obtain the condition $E \Omega E^{\top}+F \Omega F^{\top}=\Omega$, which will be key later.
The vector of operators $\hat{\boldsymbol{r}}$ was ordered so that $\hat{\boldsymbol{r}}=$ $\left(\hat{x}_{1}, \hat{p}_{1} \ldots \hat{x}_{n}, \hat{p}_{n}\right)^{\top}$. We can also consider an orthogonal symplectic matrix $S$ acting on a vector of differently ordered operators: $\hat{\boldsymbol{s}} \rightarrow S \hat{\boldsymbol{s}}$, where $\hat{\boldsymbol{s}}=\left(\hat{x}_{1} \ldots \hat{x}_{n}, \hat{p}_{1} \ldots \hat{p}_{n}\right)$. In this case, the transformation matrix takes the form [22]

$$
S=\left(\begin{array}{cc}
X & Y \\
-Y & X
\end{array}\right) \quad \text { with } \quad X Y^{\top}-Y X^{\top}=0_{n}
$$

and

$$
X X^{\top}+Y Y^{\top}=\mathbb{1}_{n} .
$$

When we use the ordering of variables $\hat{s}=$ $\left(\hat{x}_{1} \ldots \hat{x}_{n}, \hat{p}_{1} \ldots \hat{p}_{n}\right)^{\top}$, the symplectic condition is $S \omega S^{\top}=\omega$, where $\omega$ is the alternatively ordered symplectic form

$$
\omega=\left(\begin{array}{cc}
0_{n} & \mathbb{1}_{n} \\
-\mathbb{1}_{n} & 0_{n}
\end{array}\right) .
$$

Transforming between the two representations means that we can write each $2 \times 2$ submatrix of $Z$ as

$$
Z=\left(\begin{array}{ccc}
Z_{11} & \ldots & Z_{1 n} \\
\vdots & \ddots & \vdots \\
Z_{n 1} & \ldots & Z_{n n}
\end{array}\right) \quad Z_{j k}=\left(\begin{array}{cc}
x_{j k} & y_{j k} \\
-y_{j k} & x_{j k}
\end{array}\right),
$$

where $x_{j k}$ and $y_{j k}$ are the elements of matrices $X$ and $Y$ respectively. This fact will be used later.

\section{The diffusion matrix for passive coherent feedback}

We will now find an expression for the diffusion matrix in this setup. In the section titled "A general framework for Gaussian coherent feedback," the operation $Z$ was assumed to be a general symplectic. Now, we will add the extra restriction that the coherent feedback must be passive, so $Z$ is also orthogonal, meaning $Z Z^{\top}=\mathbb{1}$. This gives us the condition $E E^{\top}+F F^{\top}=\mathbb{1}$. Recall that the diffusion matrix is given by

$$
D=\Omega C \sigma_{\text {in }} C^{\top} \Omega^{\top} \text {. }
$$

We will allow the environment to be a thermal state, so $\sigma_{\text {in }}=N \mathbb{1}$. Note that we can calculate the zero-temperature diffusion matrix $D_{0}$ by setting $N=1$ and then easily modify this for nonzero temperature by multiplying by an appropriate value of $N$. Using the convention that the dimension of $\Omega$ is specified by the context, and plugging in expression (B15) for the coupling matrix under coherent feedback, we obtain

$$
D_{0}=\left(\Omega K_{l}-\Omega K_{m} E\right)\left(K_{l}^{\top} \Omega^{\top}-E^{\top} K_{m}^{\top} \Omega^{\top}\right)+\Omega K_{m} F F^{\top} K_{m}^{\top} \Omega^{\top} .
$$

Multiplying this out, and using the orthogonal condition $E E^{\top}+F F^{\top}=\mathbb{1}$ gives

$$
D_{0}=\Omega\left(K_{l} K_{l}^{\top}+K_{m} K_{m}^{\top}\right) \Omega^{\top}-\Omega K_{l} E^{\top} K_{m}^{\top} \Omega^{\top}-\Omega K_{m} E K_{l}^{\top} \Omega^{\top} .
$$

We note that $K_{l} K_{l}^{\top}=\gamma \operatorname{diag}\left(l_{1}, l_{1} \ldots l_{s}, l_{s}\right)$ and $K_{m} K_{m}^{\top}=\gamma \operatorname{diag}\left(m_{1}, m_{1} \ldots m_{s}, m_{s}\right)$. We will write

$$
\Omega\left(K_{l} K_{l}^{\top}+K_{m} K_{m}^{\top}\right) \Omega^{\top}=\gamma \operatorname{diag}\left(l_{1}+m_{1}, l_{1}+m_{1} \ldots l_{s}+m_{s}, l_{s}+m_{s}\right)=\Delta .
$$

After doing this, our expression for the zero-temperature diffusion matrix becomes

$$
D_{0}=\Delta-\Omega K_{l} E^{\top} K_{m}^{\top} \Omega^{\top}-\Omega K_{m} E K_{l}^{\top} \Omega^{\top} .
$$


We will call $J=\Omega K_{m} E K_{l}^{\top} \Omega^{\top}$, allowing us to write

$$
D_{0}=\Delta-J^{\top}-J .
$$

At this point, we will note the form of $\Omega K_{l}$ and $\Omega K_{m}$, which are given by

$$
\Omega K_{l}=\left(\begin{array}{ccc}
\Gamma_{l_{1}}^{\top} & \ldots & 0 \\
\vdots & \ddots & \vdots \\
0 & \ldots & \Gamma_{l_{s}}^{\top}
\end{array}\right)=G_{l} \text { and } \Omega K_{m}=\left(\begin{array}{ccc}
\Gamma_{m_{1}}^{\top} & \ldots & 0 \\
\vdots & \ddots & \vdots \\
0 & \ldots & \Gamma_{m_{s}}^{\top}
\end{array}\right)=G_{m} .
$$

This notation can be used to write $J=G_{m} E G_{l}^{\top}$. We also note the following easily verified relationships which we will use later:

$$
\Omega K_{m}^{\top}=-G_{m}^{\top}, \quad \Omega K_{l}^{\top}=-G_{l}^{\top}, \quad \Omega G_{m} \Omega^{\top}=G_{m}, \quad \Omega G_{l} \Omega^{\top}=G_{l} .
$$

Now, we will show that $\Omega D_{0} \Omega^{\top}=D_{0}$. This result will be useful later. First we notice that due to the diagonal form of $\Delta$, we have $\Omega^{\top} \Delta \Omega=\Delta$. Next, using the relationships in (B30) and inserting the identity $\Omega^{\top} \Omega=\mathbb{1}$, we find that

$$
\Omega J \Omega^{\top}=\Omega G_{m} E G_{l}^{\top} \Omega^{\top}=\Omega G_{m} \Omega^{\top} \Omega E \Omega^{\top} \Omega G_{l}^{\top} \Omega^{\top}=G_{m} \Omega E \Omega^{\top} G_{l}^{\top} .
$$

Writing $E$ in terms of its $2 \times 2$ submatrices gives

$$
E=\left(\begin{array}{ccc}
E_{11} & \ldots & E_{1 p} \\
\vdots & \ddots & \vdots \\
E_{q 1} & \ldots & E_{q p}
\end{array}\right), \quad \text { where } E_{j k}=\left(\begin{array}{cc}
e_{11}^{j k} & e_{12}^{j k} \\
-e_{12}^{j k} & e_{11}^{j k}
\end{array}\right)
$$

The form of the $2 \times 2$ submatrices comes from the orthogonal symplectic condition discussed in the previous section. With this form $\Omega E_{j k} \Omega^{\top}=E_{j k}$, meaning that

$$
\Omega E \Omega^{\top}=\left(\begin{array}{ccc}
\Omega E_{11} \Omega^{\top} & \ldots & \Omega E_{1 p} \Omega^{\top} \\
\vdots & \ddots & \vdots \\
\Omega E_{q 1} \Omega^{\top} & \ldots & \Omega E_{q p} \Omega^{\top}
\end{array}\right)=E
$$

Plugging $\Omega E \Omega^{\top}=E$ into (B31) gives us $\Omega J \Omega^{\top}=J$. Combining this with $\Omega \Delta \Omega^{\top}=\Delta$ yields $\Omega D_{0} \Omega^{\top}=D_{0}$, which is the desired result. This relation holds for any number of system modes.

We will now show explicitly that, for a single-system mode, $D_{0}$ is proportional to the identity. This is easily shown by noticing that for a single mode, $\Delta=\gamma\left(l_{1}+m_{1}\right) \mathbb{1}_{2}, G_{m}=$ $\Gamma_{m_{1}}^{\top}$, and $G_{l}^{\top}=\Gamma_{l_{1}}$, meaning that $J$ is just a sum over the $2 \times 2$ submatrices of $E$, multiplied by $\gamma$ :

$$
J=\Gamma_{m_{1}}^{\top} E \Gamma_{l_{1}}=\gamma \sum_{j, k}\left(\begin{array}{cc}
e_{11}^{j k} & e_{12}^{j k} \\
-e_{12}^{j k} & e_{11}^{j k}
\end{array}\right)=\left(\begin{array}{cc}
a & b \\
-b & a
\end{array}\right),
$$

where we have defined $a=\gamma \sum_{j, k} e_{11}^{j k}$ and $b=\gamma \sum_{j, k} e_{12}^{j k}$. It can now be seen that $D_{0}=\Delta-J-J^{\top}$ will be proportional to the identity.

\section{The drift matrix for passive coherent feedback}

The drift matrix of the system takes the form

$$
A=\Omega H_{S}+\frac{1}{2} \Omega C \Omega C^{\top} .
$$

We will take $H_{S}$ to be completely general and will examine the form of the matrix $\Omega C \Omega C^{\top}$ for the passive coherent feedback setup by plugging in expression (B15):

$$
\begin{aligned}
\Omega C \Omega C^{\top}= & \left(\Omega K_{l}-\Omega K_{m} E\right)\left(\Omega K_{l}^{\top}-\Omega E^{\top} K_{m}^{\top}\right) \\
& +\Omega K_{m} F \Omega F^{\top} K_{m}^{\top} .
\end{aligned}
$$

We can multiply this out and simplify using the symplectic condition $E \Omega E^{\top}+F \Omega F^{\top}=\Omega$ to obtain

$$
\begin{aligned}
\Omega C \Omega C^{\top}= & \Omega K_{l} \Omega K_{l}^{\top}+\Omega K_{m} \Omega K_{m}^{\top}-\Omega K_{l} \Omega E^{\top} K_{m}^{\top} \\
& -\Omega K_{m} E \Omega K_{l}^{\top} .
\end{aligned}
$$

First, we note that $\Omega K_{l} \Omega K_{l}^{\top}+\Omega K_{m} \Omega K_{m}^{\top}=-G_{l} G_{l}^{\top}-$ $G_{m} G_{m}^{\top}=-\Delta$, where $\Delta$ is defined by (B26) in the previous section. Next, using the properties of $G_{m}$, we observe that $\Omega K_{m} E \Omega K_{l}^{\top}=-G_{m} E G_{l}^{\top}=-J$. We can also manipulate $\Omega K_{l} \Omega E^{\top} K_{m}^{\top}$ by inserting $\mathbb{1}=\Omega \Omega^{\top}$ :

$$
\begin{aligned}
\Omega K_{l} \Omega E^{\top} K_{m}^{\top} & =G_{l} \Omega E^{\top} K_{m}=G_{l} \Omega E^{\top} \Omega \Omega^{\top} K_{m}^{\top} \\
& =G_{l} \Omega E^{\top} \Omega G_{m}^{\top} .
\end{aligned}
$$

Using the property of $E$ from (B33), we get $\Omega E^{\top} \Omega=-E^{\top}$, which means that we can write

$$
\Omega K_{l} \Omega E^{\top} K_{m}^{\top}=G_{l} \Omega E^{\top} \Omega G_{m}^{\top}=-G_{l} E^{\top} G_{m}^{\top}=-J^{\top} .
$$

Combining this with our previous results means that we can write

$$
\Omega C \Omega C^{\top}=-\Delta+J+J^{\top}=-D_{0} .
$$

This means that the drift matrix for any passive coherent feedback protocol can be written as

$$
A=\Omega H_{S}-\frac{1}{2} D_{0}
$$




\section{APPENDIX C: MULTIMODE CASE}

\section{Counterexample}

It can be verified that, in the two-mode case, for $D=\mathbb{1}$ and this choice of Hamiltonian matrix,

$$
H=\frac{1}{3}\left(\begin{array}{cccc}
1 & 0 & 1 & 0 \\
0 & 1 & 0 & -1 \\
1 & 0 & -1 & 0 \\
0 & -1 & 0 & 1
\end{array}\right),
$$

the matrix $A=\Omega H-\frac{1}{2} D$ is Hurwitz and the solution $\sigma_{\infty}$ to the Lyapunov equation $A \sigma_{\infty}+\sigma_{\infty} A^{\top}+D=0$ has minimum eigenvalue equal to 0.48 , thus showing that the $3-\mathrm{dB}$ bound does not apply to the eigenvalues of $\sigma_{\infty}$.

However, all the numerical evidence in our possession indicates that the bound will still hold on all diagonal elements of $\sigma_{\infty}$, as well as on all eigenvalues of single-mode reductions of $\sigma_{\infty}$ and, more generally, on all diagonal elements after application of any orthogonal symplectic transformation.

\section{Orthogonal symplectic bases}

Here, we will prove that, if the real vector $\boldsymbol{v}_{1}$ belongs to an orthogonal and symplectic basis $\left\{\boldsymbol{v}_{j}, j \in[1, \ldots, 2 n]\right\}$, then $\Omega \boldsymbol{v}_{1}$ is proportional to a vector of the same basis.
By hypothesis, the orthogonal matrix $R=\left(\boldsymbol{v}_{1}, \ldots, \boldsymbol{v}_{2 n}\right)$ is also symplectic. Therefore,

$$
\begin{aligned}
R \Omega R^{\top} & =\left(\begin{array}{ccc}
\boldsymbol{v}_{1}^{\top} \Omega \boldsymbol{v}_{1} & \boldsymbol{v}_{1}^{\top} \Omega \boldsymbol{v}_{2} & \ldots \\
\boldsymbol{v}_{2}^{\top} \Omega \boldsymbol{v}_{1} & \boldsymbol{v}_{2}^{\top} \Omega \boldsymbol{v}_{2} & \ldots \\
\vdots & \vdots & \ddots
\end{array}\right) \\
& =\left(\begin{array}{ccc}
0 & 1 & \cdots \\
-1 & 0 & \cdots \\
\vdots & \vdots & \ddots
\end{array}\right)=\Omega .
\end{aligned}
$$

Thence one has $\boldsymbol{v}_{j}^{\top} \Omega \boldsymbol{v}_{1}=-\delta_{j 2}: \Omega \boldsymbol{v}_{1}$ is orthogonal to all basis vectors except $\boldsymbol{v}_{2}$, and we must conclude that $\Omega \boldsymbol{v}_{1}=-\boldsymbol{v}_{2}$, Q.E.D.

In light of the above, if a covariance matrix $\sigma$ is diagonalized by an orthogonal symplectic and $\boldsymbol{v}$ is an eigenvector of $\sigma$, then $\boldsymbol{w}=\Omega \boldsymbol{v}$ must be an eigenvector too.

This argument is instrumental in showing that, for any number of system modes, an eigenvalue smaller than $1 / 2$ of a steady-state covariance matrix obtained through interferometric coherent feedback cannot correspond to the variance of a physical quadrature (defined from the original system quadratures without applying any squeezing, which would amount to cheating).
[1] H. M. Wiseman and G. J. Milburn, Quantum Measurement and Control (Cambridge University Press, Cambridge, UK, 2009).

[2] S. Lloyd, Phys. Rev. A 62, 022108 (2000).

[3] K. Jacobs, X. Wang, and H. Wiseman, New J. Phys. 16, 073036 (2014).

[4] H. I. Nurdin, M. R. James, and I. R. Petersen, Automatica 45, 1837 (2009).

[5] S. Iida, M. Yukawa, H. Yonezawa, N. Yamamoto, and A. Furusawa, IEEE Trans. Aut. Control 57, 2045 (2012).

[6] N. Yamamoto, Phys. Rev. X 4, 041029 (2014).

[7] C. W. Gardiner, Phys. Rev. Lett. 70, 2269 (1993).

[8] H. J. Carmichael, Phys. Rev. Lett. 70, 2273 (1993).

[9] V. Giovannetti and G. M. Palma, J. Phys. B: At. Mol. Opt. Phys. 45, 154003 (2012).

[10] C. C. Wanjura, M. Brunelli, and A. Nunnenkamp, Nat. Commun. 11, 3149 (2020).

[11] H. M. Wiseman and G. J. Milburn, Phys. Rev. A 49, 4110 (1994).

[12] M. Aspelmeyer, T. J. Kippenberg, and F. Marquardt, Rev. Mod. Phys. 86, 1391 (2014).

[13] K. Lalumière, B. C. Sanders, A. F. van Loo, A. Fedorov, A. Wallraff, and A. Blais, Phys. Rev. A 88, 043806 (2013).

[14] J. E. Gough and S. Wildfeuer, Phys. Rev. A 80, 042107 (2009).

[15] M. Hirose and P. Cappellaro, Nature (London) 532, 77 (2016).

[16] C. Schäfermeier, H. Kerdoncuff, U. B. Hoff, H. Fu, A. Huck, J. Bilek, G. I. Harris, W. P. Bowen, T. Gehring, and U. L. Andersen, Nat. Commun. 7, 13628 (2016).

[17] D. Risté, M. Dukalski, C. A. Watson, G. de Lange, M. J. Tiggelman, Y. M. Blanter, K. W. Lehnert, R. N. Schouten, and L. DiCarlo, Nature (London) 502, 350 (2013).
[18] A. Balouchi and K. Jacobs, Quantum Sci. Technol. 2, 025001 (2017).

[19] H. Vahlbruch, M. Mehmet, K. Danzmann, and R. Schnabel, Phys. Rev. Lett. 117, 110801 (2016).

[20] C. M. Caves, Phys. Rev. D 26, 1817 (1982).

[21] J. E. Gough, M. R. James, and H. I. Nurdin, Phys. Rev. A 81, 023804 (2010).

[22] A. Serafini, Quantum Continuous Variables: A Primer of Theoretical Methods (CRC Press, Boca Raton, FL, 2017).

[23] C. W. Gardiner and M. J. Collett, Phys. Rev. A 31, 3761 (1985).

[24] M. G. Genoni, S. Mancini, and A. Serafini, Phys. Rev. A 87, 042333 (2013).

[25] M. G. Genoni, S. Mancini, H. M. Wiseman, and A. Serafini, Phys. Rev. A 90, 063826 (2014).

[26] As a term of reference in interpreting this formula, let us point out that the maximum homodyne efficiency currently achievable is around $99 \%$ [19].

[27] Such modes clearly should not be squeezed, to avoid the unfair introduction of additional squeezing into the system; further, they should not be at a lower temperature than the original environment, since otherwise a hypothetical experimentalis could just replace the latter with one at a lower temperature, which would be a nice but highly questionable possibility in practice.

[28] S. Huang and A. Chen, Appl. Sci. 9, 3402 (2019).

[29] R. Hamerly and H. Mabuchi, Phys. Rev. Lett. 109, 173602 (2012).

[30] K. Jacobs, H. I. Nurdin, F. W. Strauch, and M. James, Phys. Rev. A 91, 043812 (2015). 